\title{
Development of a Laboratory-safe and Low-cost Detection Protocol for SARS-CoV-2 of the Coronavirus Disease 2019 (COVID-19)
}

\author{
Joungha Won, Solji Lee, Myungsun Park, Tai Young Kim, Mingu Gordon Park, Byung Yoon Choi, \\ Dongwan Kim, Hyeshik Chang, Won Do Heo, V. Narry Kim and C. Justin Lee
}

Exp Neurobiol. 2020 Apr;29(2):107-119. doi: 10.5607/en.20009. Epub 2020 Apr 30.

We would like to correct author's affiliations, add an author and an edit one sentence as shown below.

1) The corrected affiliations (switch affiliation 1 and 2) and added author are marked by bold and underlines.

Joungha Won ${ }^{1,2}$, Solji Lee ${ }^{2}$, Myungsun Park ${ }^{2}$, Tai Young Kim ${ }^{2}$, Mingu Gordon Park ${ }^{2,3}$, Byung Yoon Choi $^{4}$, Dongwan Kim ${ }^{5,6}$, Hyeshik Chang $^{5,6}$, Won Do Heo ${ }^{1}$, V. Narry Kim ${ }^{5,6}$ and C. Justin Lee ${ }^{2 *}$

${ }^{1}$ Department of Biological Sciences, Korea Advanced Institute of Science and Technology (KAIST), Daejeon 34141, ${ }^{2}$ Center for Cognition and Sociality, Cognitive Glioscience Group, Institute for Basic Science, Daejeon 34126, ${ }^{3} \mathrm{KU}-\mathrm{KIST}$ Graduate School of Converging Science and Technology, Korea University, Seoul 02841, ${ }^{4}$ Department of Otorhinolaryngology, Seoul National University Bundang Hospital, Seongnam 13620, ${ }^{5}$ Center for RNA Research, Institute for Basic Science, Seoul 08826, ${ }^{6}$ School of Biological Sciences, Seoul National University, Seoul 08826, Korea

2) In Quantitative rtPCR section (Page 110, in material and methods) we would like to correct the following sentence from; "In brief, each reaction buffer consisted of a total volume of $20 \mu$ containing $8 \boldsymbol{\mu l}$ of $\mathbf{1 0 0} \boldsymbol{\mu M}$ forward and reverse primers $(\mathbf{4} \mu \mathrm{l}$ for each primer), $2 \mu \mathrm{l}$ of cDNA, and $10 \mu$ power SYBR Green PCR Master Mix."

to;

"In brief, each reaction buffer consisted of a total volume of $20 \mu \mathrm{l}$ containing $2 \underline{\mu l}$ of $\mathbf{1 0} \mu \mathrm{M}$ forward and reverse primers $(\underline{\mathbf{1}} \mu \mathrm{l}$ for each primer), $2 \mu \mathrm{l}$ of cDNA, and $10 \mu \mathrm{l}$ power SYBR Green PCR Master Mix."

This is an Open Access article distributed under the terms of the Creative Commons Attribution Non-Commercial License (http://creativecommons.org/licenses/by-nc/4.o) which permits unrestricted non-commercial use, distribution, and reproduction in any medium, provided the original work is properly cited. 